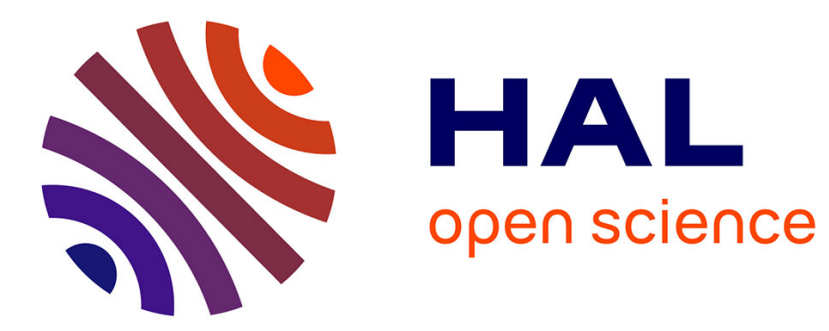

\title{
Molecular Genetics of the Fibrillinopathies
}

Mélodie Aubart, Louise Benarroch, Pauline Arnaud, Gwenaelle

Collod-Beroud, Guillaume Jondeau, Catherine Boileau

\section{To cite this version:}

Mélodie Aubart, Louise Benarroch, Pauline Arnaud, Gwenaelle Collod-Beroud, Guillaume Jondeau, et al.. Molecular Genetics of the Fibrillinopathies. eLS, 2016, 10.1002/9780470015902.a0025314 . hal-01682320

\section{HAL Id: hal-01682320 \\ https://hal-amu.archives-ouvertes.fr/hal-01682320}

Submitted on 12 Jan 2018

HAL is a multi-disciplinary open access archive for the deposit and dissemination of scientific research documents, whether they are published or not. The documents may come from teaching and research institutions in France or abroad, or from public or private research centers.
L'archive ouverte pluridisciplinaire $\mathbf{H A L}$, est destinée au dépôt et à la diffusion de documents scientifiques de niveau recherche, publiés ou non, émanant des établissements d'enseignement et de recherche français ou étrangers, des laboratoires publics ou privés. 


\section{Molecular Genetics of the Fibrillinopathies}

Mélodie Aubart ${ }^{\star}$, Laboratory for Vascular Translational Science, INSERM U1148, Paris, France

Louise Benarroch, Laboratory for Vascular Translational Science, INSERM U1148, Paris, France

Pauline Arnaud, Laboratory for Vascular Translational Science, INSERM U1148, Paris, France and Département de Génétique, Centre Hospitalier Universitaire Xavier Bichat, Assistance Publique Hôpitaux de Paris, Paris, France

Gwenaëlle Collod-Béroud, INSERM UMR_S910, Marseille, France

Guillaume Jondeau, National Reference Centre for Marfan Syndrome and Related Disorders, Service de Cardiologie, Centre Hospitalier Universitaire Xavier Bichat, Assistance Publique Hôpitaux de Paris, Paris, France

Catherine Boileau, Laboratory for Vascular Translational Science, INSERM U1148, Paris, France and Département de Génétique, Centre Hospitalier Universitaire Xavier Bichat, Assistance Publique Hôpitaux de Paris, Paris, France

^Presently at Service de Neuropédiatrie, Hôpital Necker-Enfants malades, Assistance Publique Hôpitaux de Paris, Paris, France

Fibrillins are major glycoprotein components of microfibrils in the extracellular matrix. They are polymerised in a complex beads-on-a-string appearance and form isolated aggregates or are closely associated with elastin in elastic fibres. Fibrillins are critical actors of the biomechanical function of connective tissue and regulators of the bioavailability of signalling molecules, especially TGF- $\beta$. Mutations in the FBN1 and FBN2 genes are associated with inherited diseases now termed fibrillinopathies. These diseases represent a wide spectrum of disorders including Marfan and neonatal Marfan syndrome, acromelic dysplasias, progeroid-marfanoid syndrome, the stiff skin syndrome and congenital contractural arachnodactyly. Clinical symptoms are varied, thus highlighting the importance of fibrillins in tissue development and integrity. The identification of over $\mathbf{3 0 0 0}$ mutations in FBN1 and over

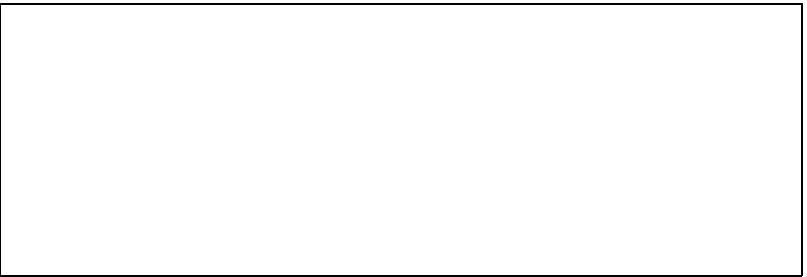

100 in FBN2 has provided only a few instances of genotype-phenotype correlations but have provided clues in the functional role of fibrillin domains.

\section{Introduction}

Fibrillins are the major components of the connective tissue that were identified in the late 1980s. Quickly thereafter, mutations were identified in the gene-encoding fibrillin-1 (FBN1) in patients with Marfan syndrome (MFS) (Robinson, 2006), the founding member of inherited connective disorders. Through the past 25 years, mutations in the fibrillin genes have been identified in various diseases now referred to as fibrillinopathies. These diseases present a very wide spectrum of clinical features, overlapping in some cases or completely diverging mirror images of one another in other cases. The description of these diseases and the development of animal models have provided major insight not only into fibrillin biology and tissue homeostasis but also into the pathogenic mechanisms of these diseases.

\section{Fibrillins}

FBN1 is a 350-kDa extracellular glycoprotein, well conserved from jellyfish to man. FBN1 is the major component of microfibrils in the extracellular matrix (ECM). It is an ubiquitous protein, 
predominant in elastic and nonelastic connective tissues. FBN1 has a multimodular structure which contains approximately $14 \%$ cysteines of which one-third are in the free reactive sufhydril form (Sakai et al., 1991). Fibrillin 2 (FBN2) and Fibrillin 3 (FBN3) are similar to FBN1 with a conserved modular domain organisation, but amino-acid homology is 61-69\%. Fibrillin molecules are associated in microfibrils which display beads-on-a-string appearance with a 56-nm periodicity. These microfibrils are found either as isolated aggregates or associate with tropoelastin during elastic fibrillogenesis to form mature elastic fibres (Jensen et al., 2012). Fibrillins are not only critical actors of the biomechanical function of connective tissue but also regulators of the bioavailability of various molecules involved in signalling pathways, notably TGF- $\beta$ (transforming growth factor).

\section{Domains}

Human FBN1 consists of 2871 amino acids, while FBN2 and FBN3 contain 2912 and 2809, respectively. The three fibrillins have a modular structure of $46 / 47$ epidermal growth factor-like (EGF-like) domains, with 42/43 calcium-binding types. These are also found in numerous other multidomain proteins (over 1000 according to uniprot (www.uniprot.org)) in particular in proteins constituting or regulating the ECM and serum proteins (complement, coagulation factors). EGF-like domains contain six highly conserved cysteine residues with three disulfide bonds (1-3, 2-4 and 5-6) playing a major role in stabilisation of the polymeric final structure (Downing et al., 1996). The majority of these EGF-like domains have a consensus sequence for calcium binding (cb) in the $N$-terminal pocket of the domain (Handford et al., 1991). Calcium binding stabilises contiguous cb-EGF domains into a rigid linear structure. The rod-shaped structure (in the presence of calcium) is necessary for the assembly of microfibrils, interactions with other proteins and protection from proteolysis.

Other domains of fibrillins are the TGF-binding protein motifs (TB, also known as eight cysteine motifs, 8-Cys). They are specific of fibrillins and latent TGF- $\beta$-binding-proteins (LTBPs). They contain an unusual contiguous internal cluster of three cysteine residues within an $\alpha$-helical region. The cysteine residues form intradomain disulfide bonds in a 1-3, 2-6, 4-7 and 5-8 association (Lack et al., 2003). These domains are repeated seven times in the fibrillins and three times in the LTBPs. Unlike fibrillins, the third TB motif of LTBPs contains a Phe-Pro insertion that renders the 2,6 disulfide bond more easily accessible for interactions with the TGF- $\beta 1$ propeptide. It allows the formation of a complex with the TGF- $\beta 1$ propeptide (latency associated protein, LAP). Other specificities can also be found between fibrillin and LTBP TB domains such as hydrophobic patches and negatively charged residues with a large electrostatic surface potential.

Hybrid domains are also unique to fibrillins (x2) and LTBPs (x1). They are derived through evolution from consensus TB domains in their $N$-terminal part and consensus cbEGF-like domains in their $C$-terminal part. They also contain $8 / 9$ cysteines forming intramolecular disulfide bonds important for stabilisation. The $N$-terminal end harbours the 27 amino-acids signal peptide followed by the so-called 29 residue $\mathrm{N}$-terminal region that contains basic residues and a putative furin cleavage site. The 184 amino-acid $C$-terminal region is located after the last cbEGF-like domain. This $C$-terminal domain contains, before the cleavage site of two conserved cysteine residues, a sequence with some homology to the $C$-terminal domain of members of the fibulin family (Giltay et al., 1999).

Differences between fibrillins are found between amino acids 402 and 446: a prolin-rich domain in FBN1, a glycin-rich domain in FBN2 and a proline- and glycine-rich domain in FBN3. These unique regions could be associated with a yet unknown critical function. Finally, fibrillins also display integrin-binding sequences and glycosylation sites that differ between the three forms.

\section{Expression}

Fibrillins are quite different in their expression patterns. FBN1 has an ubiquitous expression showing a spectrum from especially high levels in skin fibroblasts to quite low in central nervous system. Its expression is lifelong from development to postnatal growth and adult (Quondamatteo et al., 2002; Hubmacher et al., 2006). Conversely, FBN2 is mainly expressed during development and tissue remodelling and also in the placenta. Thereafter, it is weakly expressed almost exclusively in fibroblasts. Finally, $F B N 3$ is also moderately expressed and at low levels in the connective tissues of a few organs (lung, kidney and glands such as the thyroid).

\section{Role in the ECM}

Microfibrils were described for the first time in 1962 (Low, 1962) as independant extracellular structural units variable in thickness. They are abundant not only in elastic tissues (aorta, skin, etc.) in association with tropoelastin-forming mature elastic fibres (Ramirez and Sakai, 2010) but also in dynamic tissues that do not express elastin such as the ciliary zonule, maintaining the ocular lens in a dynamic suspension.

FBN1 was first reported in 1986 and described as long flexible molecules that are major structural components of 12-20 nm diameter microfibrils. Thus, during the first decades of their identification and description, fibrillins (and microfibrils) were reported as a supporting structure (Ramirez and Sakai, 2010). The first descriptions of mutations in TGFBR2 (encoding the TGF- $\beta$ receptor, type 2) in MS in 2004 (Mizuguchi et al., 2004) led to consider the microfibril scaffold as a niche for growth factors (in particular TGF- $\beta$ ) and mechanosensation (Sengle and Sakai, 2015). However, structural and signalling roles of microfibrils are stage-specific, tissue-specific and fibrillin-type specific.

\section{Fibrillins as a support structure}

FBN1 proteins are organised in a head to tail arrangement and also associate laterally at the cell surface (Charbonneau et al., 2010). However, FBN1 microfibrils are highly complex polymers and the molecular basis of assembly is not completely understood. Receptors on cell surfaces are predicted to have a key role in the process, as for fibronectin assembly, especially some integrins $(\alpha 5 \beta 3$ and $\alpha v \beta 3)$ and heparan sulfate proteoglycans. The 
initial step in assembly is the intermolecular disulfide bonding, especially at the $N$-terminus. Then, $N$ - and $C$-terminal regions, downstream of the furin site, could interact and their association could drive linear accretion of furin-processed FBN1 molecules in the extracellular space-forming homotypic FBN1 or heterotypic FBN1/FBN2 microfibrils (Kielty et al., 2005). However, the $\mathrm{N}$ - and $\mathrm{C}$-terminal homotypic interactions are critical in lateral assembly with bonding through transglutaminase cross-linking.

Microfibrils provide the extracellular scaffold that guides tropoelastin alignment and cross-linking (Kielty et al., 2005). It allows adaptation of elastic fibres to different tissue-specific organisations with different mechanical requirements. For example, in the arterial wall, elastic fibres must be organised as concentric rings that support thoracic aorta compliance.

In this structure, fibrillins are synthesised by vascular smooth muscle cells (vSMCs) during development. Afterwards, vSMC phenotype changes from a role in biosynthesis to contraction and maturation of the ECM. Thus, in the thoracic aorta, elasticity is supported by the mechanical properties of elastin microfibrills and vSMCs. Conversely in the dermis, microfibrils and elastic fibres form a loose meshwork (Ramirez and Sakai, 2010).

\section{Fibrillins as a reservoir of cytokines}

The ECM contains microfibrils and microfibril-associated proteins (MAGPs) such as MAGP1 and MAGP2 (Gibson et al., 1996), and also decorins, versican, perlecan, fibulins, elastin, ADAMTS-like proteins and so on. Growth factors are also found, and their metabolism is regulated through sequestration and release from various ECM proteins to which they are bound in specific regions. In this way, the ECM represents a reservoir where growth factors are nested. Some of these interactions have been described as playing a major role in the pathophysiology of thoracic aortic aneurysm (TAA) (Regalado and Millewicz, 2015), MAGP2 (also known as MFAP5) and TGF- $\beta$. Indeed, mutations in the genes encoding these proteins (TGFB2, Boileau et al., 2012 and MFAP5, Barbier et al., 2014) have been shown to be involved in familial TAA and in genes encoding other downstream actors of the signalling cascade (TGFBR1, Loeys et al., 2005; TGFBR2, Mizuguchi et al., 2004 and SMAD3, Van de Laar et al., 2011). TGF- $\beta$ molecules are produced as inactive latent complexes in which active TGF- $\beta$ is enveloped by its propeptide (LAP) and bound to FBN1 through LTBP. Release of active TGF- $\beta$ in the ECM leads to the activation of TGF- $\beta$ receptors (homo or heterodimeric TGFBR1/2) and phosphorylation of receptor-regulated SMADs (especially SMAD2 and SMAD3). These SMAD proteins form a complex with the common-mediator SMAD (co-SMAD) to transduce extracellular signals to the nucleus to activate targeted gene transcription. Many genes are regulated by SMAD signalling, especially those encoding ECM components and regulators (collagens, metalloproteases MMPs and their tissue-inhibitors TIMPs, etc.). TGF- $\beta$ receptors also activate other signalling pathways, as the ERK and JNK cascade. It is now widely accepted that along with structural defects, MFS and TAA pathophysiology also includes excess active TGF- $\beta$ signalling and dysregulation between the SMAD and ERK and JNK pathways (Regalado and Millewicz, 2015).

\section{FBN1 Mutation-Associated Disorders}

\section{The Marfan syndrome (MFS)}

MFS (OMIM \#154700) is a rare connective tissue disorder with an autosomal dominant transmission. Its prevalence is estimated around 1/5000 individuals. It is a multisystem disease associating not only cardiovascular, skeletal and ocular features but also skin, lung and dura. Around $25 \%$ of the MFS cases are sporadic (owing to de novo mutations). MFS is characterised by great clinical variability and in the age of appearance of the symptoms as in their number or severity.

\section{Clinical features}

TAA is the main cardiovascular feature. It can lead to dissection of the aortic wall, a major life-threatening event. The aortic dilatation is predominant at the root of the aorta called sinus of Valsalva level (Figure 1a). Evolution of TAA is nonlinear and thus it is not possible to predict the dilatation rate or the risk of dissection (Détaint et al., 2010). Hypertension, bicuspid aortic valve and pregnancy can contribute to increase the risk of dilatation and dissection. As there is no curative treatment, three methods are currently used to control this cardiovascular risk: life-long $\beta$-blocker therapy, prevention of heavy lifting and preventive aortic replacement surgery (Samadi et al., 2012) when aortic diameter reaches $50 \mathrm{~mm}$ (compared to $55 \mathrm{~mm}$ in nongenetic TAA). Acute aortic events are rare before the age of 20 years but increase with age with $74 \%$ of patients affected at 60 years. Aortic risk is described as higher in males than females. Mitral valve prolapse is another important cardiovascular feature and can be complicated by mitral valve insufficiency. Arterial features can be completed by arterial tortuosity (Morris et al., 2011), but no clear association of MFS with adverse medium-size artery events (aneurysm or dissection) has been reported, unlike other forms of familial TAA.

The other predominant system involved in MFS is the eye with an elongated eyeball and hypermobility of the ciliary zonule leading to ectopia lentis (Figure 1b), myopia and flat cornea. Lens dislocation or retinal detachment can then occur, possibly leading to blindness.

Other connective tissues can be altered in MFS as the skeleton with occurrence of arachnodactyly (Figure 1c), dolichostenomelia, anterior chest deformity, scoliosis, tall stature, protrusio acetabulae, hypermobility and pes planus and also the skin with striae and herniae, the lung with spontaneous pneumothorax and the dura with dural ectasia (lumbosacral meningocele).

It should be noted that a particularly severe form of MFS was described in 1985 and later named neonatal MFS (Stheneur et al., 2011). This very early-onset disease (diagnosed between birth and the third month of life) displays severe cardiovascular features (TAA and mitral and tricuspid valve dysfunction), pulmonary emphysema, joint contractures, crumpled ears and cutis laxa. More classical symptoms such as arachnodactyly and ectopia lentis are also found (Hennekam, 2005). The most recent 


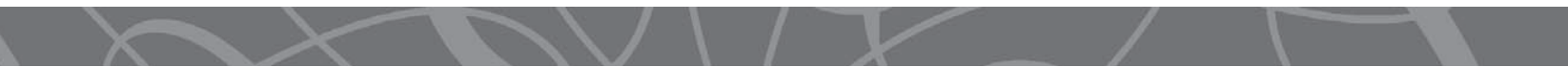

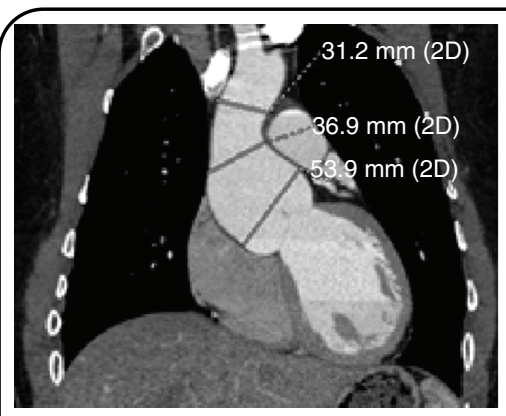

(a)

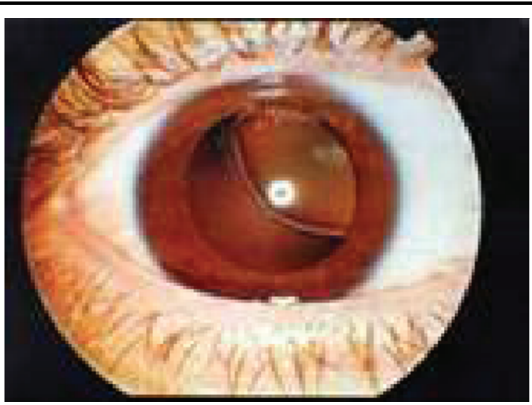

(b)

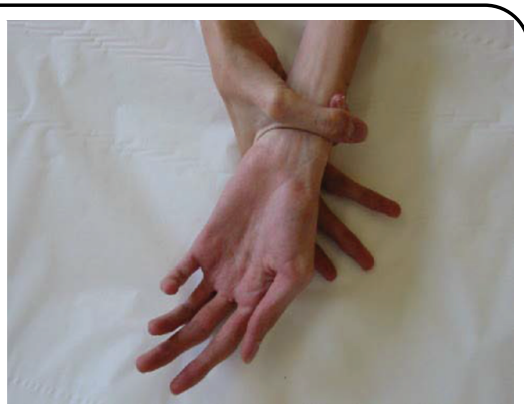

(c)

Figure 1 Clinical features of Marfan syndrome. (a) Cardiac ultrasound showing aortic dilatation at the sinus of Valsalva level. (b) Ectopia lentis. (c) Arachnodactyly (positive wrist sign).

review of 60 children diagnosed before the age of 1 showed a mortality of $2 / 3$, mostly due to valvular dysfunction or diaphragmatic herniae (Stheneur et al., 2011).

\section{Clinical classification}

The first case of MFS was described by a French pediatrician, Antoine Bernard-Jean Marfan (1858-1942), in 1896. Gabrielle $\mathrm{P}$ was a 5-year-old child with 'spider-like' legs and long arms and fingers leading to the first definition of dolichostenomelia. The first association of this condition with ectopia lentis was in 1914 and with TAA in 1942. Finally, the first clinical review of 50 families (105 individuals) was published by McKusick (1955).

First diagnostic criteria were defined in 1986 at the 7th International Congress of Human Genetics in Berlin (Berlin classification) before the emergence of molecular biology (Beighton et al., 1988) (Figure 2). Then, the nosology was revised through the Ghent criteria in 1996 (De Paepe et al., 1996) that allowed a more precise definition of MFS that integrated molecular data (Figure 2). More recently, familial investigations led to description of families with isolated ectopia lentis or to relatives with no or minor symptoms. Thus, the Ghent criteria were described as effective for their specificity, but with a sensitivity of only $80 \%$ (Faivre et al., 2009). Therefore, a revision of the Ghent criteria was proposed with other combinations of the different criteria, giving more weightage to TAA, ectopia lentis and family history (specific features) that are associated with a global 'systemic score' (Figure 2) (Loeys et al., 2010b).

\section{Molecular biology}

The FBN1 gene comprises 65 exons, a $3^{\prime}$ UTR region and a promoter with three alternatively spliced upstream exons, initially termed exons B, A and C, each of which can be spliced to the first coding exon (exon 1, previously termed exon $\mathrm{M}$ ), with a strong bias for the use of exon A (Corson et al., 1993). There is no TATA-box in the promoter but multiple Sp1 transcription binding sites. It should be noted that FBN1 transcription is on the reverse sense.

The first evidence that FBN1 mutations cause MFS came from the finding of a recurrent de novo mutation in two unrelated probands (Dietz et al., 1991). Since 1991, it has been shown that about $90 \%$ of MFS cases are related to FBN1 mutation. These are loss-of-function mutations (splicing mutations, exonic deletion or localised insertion/deletion in or out-of-frame) and missense mutations. There is no hot spot for mutation in the gene (Figure 3). To date, no strong genotype-phenotype correlation has been identified but that of neonatal MFS and missense mutations in exons 24-32 (Collod-Béroud et al., 1997). The study of more than 1000 probands (Faivre et al., 2007) showed that missense mutations involving cysteines led to more frequent ectopia lentis and that missense mutations between exons 24 and 32 led not only to neonatal MFS but also to early-onset and severe MFS.

Two pathophysiological mechanisms have been involved for FBN1 mutations in MFS: negative dominance and haploinsufficiency. Haploinsufficiency was demonstrated by FBN1 expression studies in skin fibroblasts of 80 MFS with nonsense or splicing mutations or exonic deletions (Aubart et al., 2015). Under this model, a correlation between FBN1 expression in skin fibroblasts and some MFS features, especially ectopia lentis, was found. One of the explanations of this observation is probably that the ciliary zonule, holding the lens in dynamic suspension in the eye, is one of the most FBN1-rich structures of the organism.

Regarding missense mutations, it should be noted that some domains contain no proven pathogenic mutation (according to FBN1 mutations database, www.umd.be/FBN1/) and our own results (over 1300 mutations identified to date), such as the proline-rich domain and the $\mathrm{COOH}$ unique region. Other domains contain only a few missense mutations like the signal peptide and the $\mathrm{NH}_{2}$ unique region or the first cbEGF-like module (Figure 3). This observation could highlight an evolutionary requirement for this region with high lethality of mutations. Another hypothesis could be a tissue and/or function specificity of the domain which could lead to another unknown phenotype. Other domains contain only a few missense mutations such as the signal peptide, the NH2 unique region, the first cbEGF-like module and fifth TB module in MFS patients. Finally, even if missense mutations in exons 24-32 (third TB and eleventh to eighteenth cbEGF-like domains) are especially associated with a severe or neonatal MFS phenotype, mutations in this region are also found in classic adult forms of MFS (Faivre et al., 2007). 

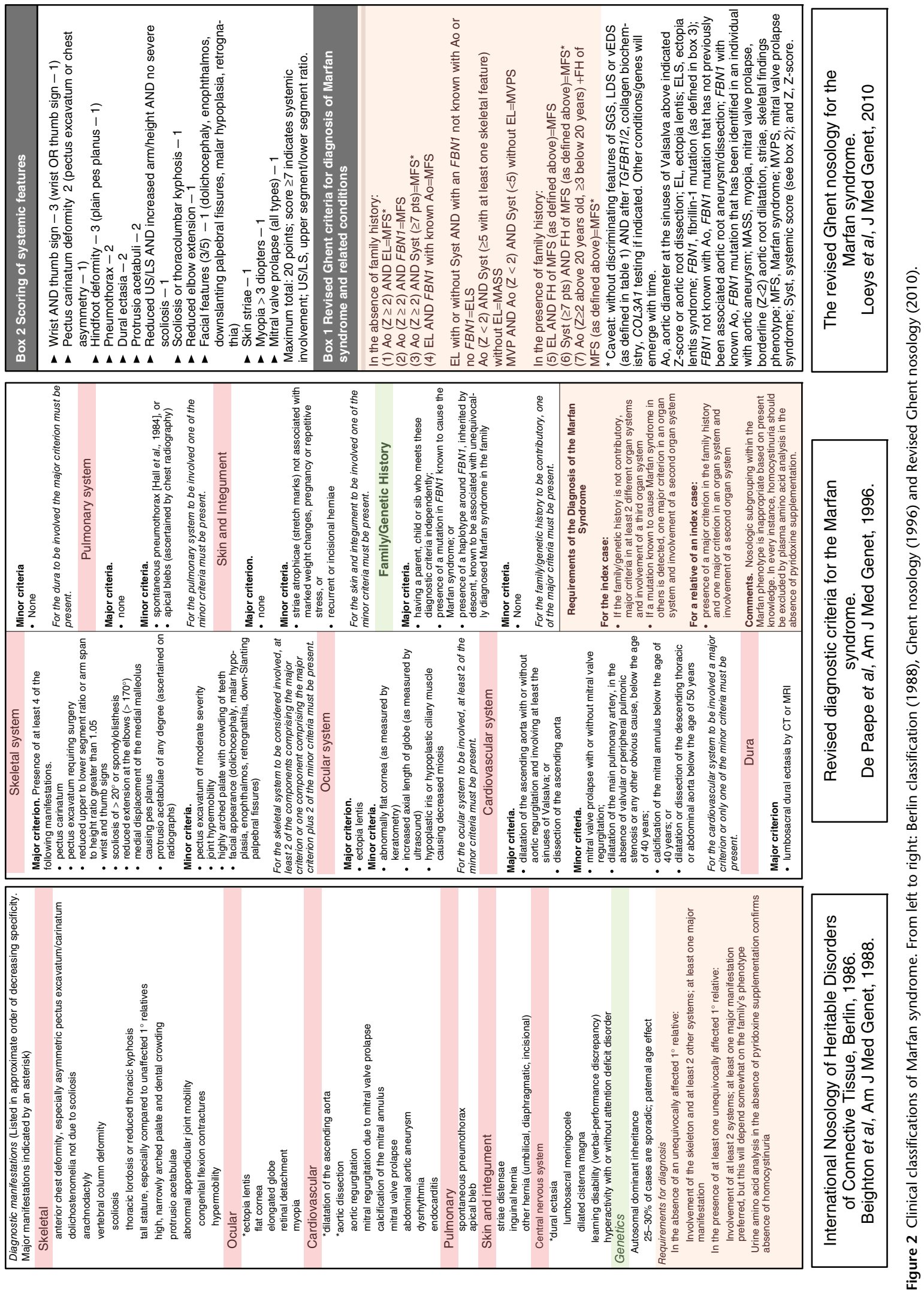


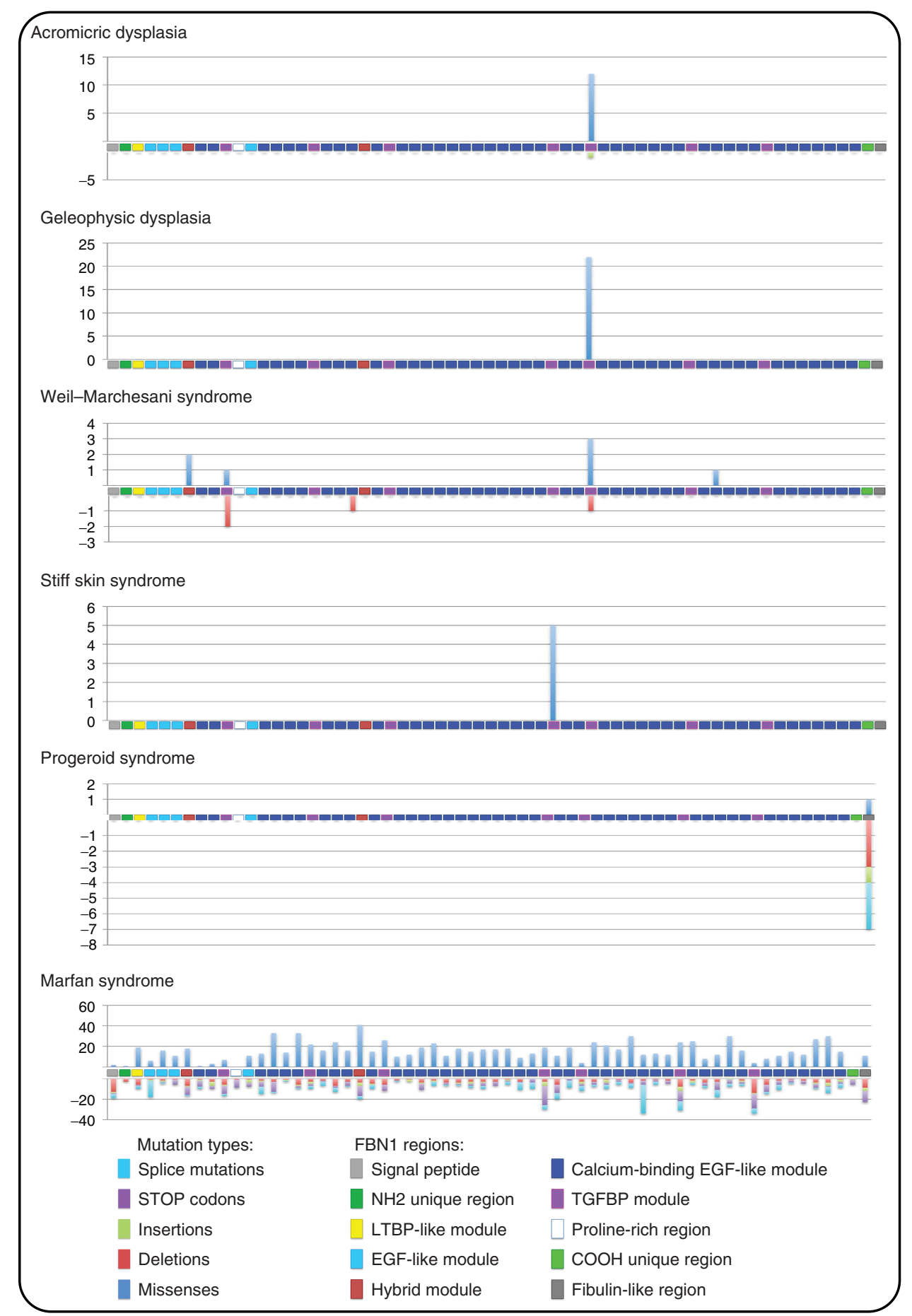

Figure 3 Hot spot for mutations in the FBN1 gene and fibrillinopathies: acromiric dysplasia, geleophysic dysplasia, Weil-Marchesani syndrome, stiff skin syndrome, progeroid syndrome and Marfan syndrome. 


\section{FBN1 mutations: the acromelic dysplasia group}

The acromelic dysplasia group is characterised by short stature, short hands and feet, stiff joint and muscular build (Le Goff and Cormier-Daire, 2012). Four disorders are described in this group: Weil-Marchesani syndrome (WMS, \#608328), geleophysic dysplasia (GD, \#231050), acromicric dysplasia (AD, \#102370) and Myhre syndrome (\#139210). They are distinguished by their pattern of inheritance and some of their clinical features. WMS is the only syndrome with eye disease: dislocated lens and microspherophakia causing severe myopia. GD is the most severe of these syndromes because of a progressive cardiac valvular thickening leading to early death. AD and Myrhe syndrome are characterised by specific facial features (round face, full lips, well-defined eyebrows, long eyelashes, bulbous nose with anteverted nostrils, long philtrum and thick lips in $\mathrm{AD}$, short palpebral fissures, maxillary hypoplasia, prognathism and short philtrum in Myrhe syndrome). Myrhe syndrome also induces deafness and intellectual disability.

The paradox of the antagonism and overlap of part of these symptoms with those of MFS found an answer in 2011 with the first description of heterozygous missense mutations in FBN1 for acromicric and GD by Le Goff et al. (2011) in a total of $29 \mathrm{AD}$ or GD cases. All the mutations have been found in the fifth TB domain (exons 41 and 42). Paradoxically, TGF- $\beta$ levels have been quantified in patient's skin fibroblasts and their cultured medium and a high enhanced signal was found. This report was completed by case reports of FBN1 mutation carriers with WMS (short stature, brachydactyly) and also overlapping MFS symptoms such as thoracic aortic dilatation or dissection, striae, contractures and ectopia lentis. Regarding Myrhe syndrome, it is not related to FBN1 mutations but to SMAD4 mutations (Le Goff and Cormier-Daire, 2012). This is a further argument supporting the involvement of TGF- $\beta$ signalling in the pathogenesis of acromelic dysplasia and some TGFB1 mutations reported in Camurati-Engelmann disease (an autosomal dominant bone dysplasia resulting in increased bone formation, cortical thickening of the diaphyses of the long bones and marfanoid habitus) (Janssens et al., 2006).

\section{Stiff skin syndrome}

The stiff skin syndrome (SSS, \#184900) was first described by Esterly and McKusick in 1971 who reported four patients presenting localised areas of 'stony-hard' skin, limitation of joint mobility and mild hirsutism. This condition was reported in other patients with skin abnormalities essentially located on thighs, buttocks and hips (areas with abundant fascia), even in some cases on the proximal arm and shoulder girdle. Focal lipodystrophy and muscle weakness as well as bone hypoplasia related to chest and member deformities and compromised lung function from severe chest limitation have also been reported. Furthermore, secondary esophageal dysmotility and gastroesophageal reflux have been reported in adults (Loeys et al., 2010a,b). Contrary to systemic sclerosis, SSS displays no visceral involvement, no Raynaud phenomenon and no immunological component. Overall, less than 70 patients have been reported worldwide. The syndrome usually appears between birth and the first 6 years of age. Although mostly observed in sporadic cases, a few multiplex families are in favour of an autosomal dominant inheritance pattern. Histologically, an altered subcutis with thickened collagen bundles oriented horizontally in a woven configuration is observed, while the dermis presents normal collagen configuration and normal elastic fibre distribution (McCalmont and Gilliam, 2012).

A spontaneous mouse model for systemic sclerosis has been known since 1967: the Tsk1 mouse (Siracusa et al., 1996). While homozygotes die early in utero, heterozygotes display excessive growth of connective tissue, including loose connective tissue, cartilage and endochrondral and membrane bone. The phenotype is due to a large in-frame duplication in the Fbn1 gene (see Section titled 'Animal Models'). Some similarities between the Tskl phenotype and SSS led to sequencing of the FBN1 gene in SSS patients and identified all missense mutations located within exons 37 and 38, encoding the $N$ - and $C$-terminal portions of TB4 (Loeys et al., 2010a, 2010b). Interestingly, only five mutations have been reported to date (Figure 3). They are all missense mutations. Four nucleotide substitutions lead to the loss of a cysteine residue (implicated in a disulfide bond) or gain of a cysteine residue (that could alter proper disulfide bond formation): p.Cys1564Ser (c.4691G>C), p.Trp1570Cys (c.4710G $>$ C or c. $4710 \mathrm{G}>\mathrm{T}$ ) and p.Cys1577Gly (c.4729T $>\mathrm{G})$. Interestingly, p.Cys1564Ser (c.4691G>A) was also found in an Australian MFS patient (Biggin et al., 2004), and at the same position, p.Cys1564Phe (c.4691G > T) was reported in an Italian MFS patient. The fifth FBN1 mutation found in an SSS patient is p.Gly1594Asp (c.4781G>A). Again, mutations had already been found at this position p.Gly1594Val (c.4781G $>$ T) in two subjects: an English proband with isolated ectopia lentis (Howarth et al., 2007) and a Japanese patient presenting features of the MFS spectrum (Sakai et al., 2006). All these observations thus preclude the establishment of a direct genotype/phenotype correlation.

\section{Progeroid fibrillinopathy}

The neonatal onset of progeroid (\#264090) and MFS features is now well documented (Jacquinet et al., 2014). The clinical presentation is very variable and can also present lipodystrophy and craniosynostosis. Contrary to other forms of the neonatal progeroid spectrum, survival is good with reported adult patients. This Marfanoid-progeroid syndrome is related with mutations that cluster on exon 64 of the FBN1 gene (Figure 3). Apart from a single missense mutation, all lead to a premature stop codon beyond amino acid 2693, with or without skipping of exon 64 . Therefore, it seems reasonable to conclude that there is a genotype/phenotype correlation and to formulate various pathogenic mechanisms (notably alteration or loss of the furin cleavage site encoded by exon 64). However, careful examination of the FBN1 mutation database reveals that comparable mutations with comparable predicted effect (premature termination) have been reported in patients with MFS and no progeroid feature. Therefore, further research is necessary to understand the diversity of clinical features related with PTC mutations in this region of the FBN1 gene. 


\section{FBN2-Mutation-Associated Disorders: Congenital Contractural Arachnodactyly (CCA) or Beals Syndromes}

Beals syndrome (also known as CCA or distal arthrogryposis type 9, \#121050) is a rare autosomal dominant disorder. For a long time, it was considered to be part of the MFS clinical spectrum until the discovery of FBN2 mutations in this syndrome showed that it was another disorder (Putnam et al., 1995; Wang et al., 1996). FBN2-mutation carriers were described with only-onset symptoms of arachnodactyly, progressive scoliosis, crumpled ears, camptodactyly, general muscle weakness and congenital contractures of all type of joints, improving with age. Other MFS symptoms are rare. Penetrance of the skeletal symptoms has been evaluated around $80 \%$ (Callewaert et al., 2009). Associated cardiovascular symptoms are rare with $13 \%$ of TAA and $1 \%$ of mitral valve prolapse. Rare congenital heart defects are also described. Until now, ectopia lentis is absent from the clinical reports. The spontaneous improvement described in joint contracture may be due to the difference in $F B N 1$ and $F B N 2$ expression during development: their expressions are quite similar during foetal life, but $F B N 2$ expression strongly decreases during postnatal development (Quondamatteo et al., 2002).

Molecular analysis of FBN2 mutations revealed that they are exclusively missense or in-frame splicing mutations between exons 23 and 34, corresponding with cbEGF-like domains of FBN2. They suggest a gain-of-function or a dominant negative mechanism.

Finally, we can note that molecular biology, by discovery of FBN2 mutations, winked at the history of medicine. Indeed, many experts retrospectively think that Gabrielle P, the first child to be described by Antoine Marfan as MFS, should have a CCA more than an MFS.

\section{Animal Models}

Apart from a spontaneous bovine MFS-like animal with a missense mutation in the Fbnl gene (Singleton et al., 2005), all animal models available are those that were developed to understand the pathogenic mechanisms of the FBN1 mutations identified in man and subsequently develop appropriate therapeutic strategies. Therefore, through the past decades, several mice strains have been generated to understand FBN1 and FBN2 functions, their part in development and their involvement in the onset of human diseases (Table 1).

The first two mice lines were engineered by Pereira et al. to mimic the dominant negative effect of Fbnl mutations seen in patients with MFS: $\mathrm{mg} \Delta$ and $\mathrm{mgR}$ (Pereira et al., 1999; Hubmacher et al., 2006). In mg $\Delta$ mice, exons 19-24 of Fbnl gene were replaced by a neomycin-resistance (neoR) expression cassette, causing reduced expression of $\mathrm{Fbnl}$ gene (10\% of the wild-type level). In $\mathrm{mgR}$ mice, a neoR cassette was inserted between exons 18 and 19. The heterozygous mice from both strains $(\mathrm{mg} \Delta /+$ and $\mathrm{mgR} /+)$ are similar to wild-type littermates.
However, the homozygous mice $(\mathrm{mg} \Delta / \mathrm{mg} \Delta$ ) died 3-4 weeks after birth owing to cardiovascular complications (rupture of the aortic wall at the root of the aorta). No skeletal abnormalities were noticed in these mice. Histopathological analysis showed reduced Fbn1 microfibril networks and resembled what was seen in MFS patients. The homozygous $\mathrm{mgR}$ mice $(\mathrm{mgR} / \mathrm{mgR})$ display reduced expression of $F b n 1$ gene (20-25\% of the wild-type level). They died 3-4 months after birth, owing to cardiovascular complications. They had skeletal signs as well, including kyphosis and ribs overgrowth and aortic medial calcification. However, as the neoR cassette interfered with Fbnl gene expression, the pathogenic mechanism in these mice did not recapitulate the effect of the dominant negative mutations found in patients (Pereira et al., 1999). In 2010, the same team created a variant of the mg $\Delta$ mouse model in which the neoR cassette was removed, using Cre-Lox recombination (Hubmacher et al., 2006). Compared to $\mathrm{mg} \Delta$ mice, heterozygous mice $\left(\mathrm{mg} \Delta^{\text {loxPneo }} /+\right)$ presented some MFS features including cardiovascular, skeletal and pulmonary defects. Indeed, $\mathrm{mg} \Delta^{\text {loxPneo}} /+$ showed dilatation of the aortic wall as well as kyphosis and emphysema. Moreover, this mutation was tested in two different genetic backgrounds, 129/Sv and C57BL/6. Mice from each strain differed significantly in the phenotype. For instance, the onset of the disease was earlier in $129 / \mathrm{Sv}$ than on the C57BL/6 background, suggesting the existence of genetic modifiers in MFS. These mice and their differences highlight two mechanisms, haploinsufficiency and negative dominant effect, that could initiate the pathogenesis of MFS. To elucidate which mechanism is implicated in the onset of MFS, Judge et al. (2004) engineered two transgenic mice, $F b n 1^{\mathrm{C} 1663 \mathrm{R}}$ and $\mathrm{Fbnl}{ }^{\mathrm{C} 1039 \mathrm{G}}$, both implicating a cysteine residue, the most often alteration found in MFS patients. Fbn $1^{\mathrm{C} 1663 \mathrm{R}}$ mice were created using a human FBNl transgene with the p.Cys1663Arg (C1663R) mutation but showed no abnormality. On the other hand, $F b n l^{\mathrm{Cl} 1039 \mathrm{G}}$ heterozygous knock-in mice $\left(F b n l^{\mathrm{C} 1039 \mathrm{G} /+}\right)$ displayed a phenotype similar to MFS patients. In man, p.Cys1039Tyr (the corresponding mutation of mouse p.Cys1039Gly (C1039G)) causes the classical form of MFS. $\mathrm{Fbn1} 1^{\mathrm{C} 1039 \mathrm{G}} /+$ mice had a normal life span and did not die because of aorta dissection. After 2 months of life, a progressive deterioration of the media was observed including elastic fibre fragmentation, diminished FBN1 network and thickened aortic wall. These mice presented skeletal abnormalities, kyphosis and rib overgrowth. Moreover, introduction of a wild-type human FBN1 transgene rescued the aortic phenotype. These observations were consistent with the idea that haploinsufficiency of the WT FBN1 is the primary determinant of failed microfibril assembly, rather than a dominant negative effect of the mutant proteins. The $F b n 1^{\mathrm{C} 1039 \mathrm{G}}$ mice were also used in a study regarding the effect of Losartan, an angiotensin II type 1 receptor blocker, as a potential treatment strategy for both cardiovascular and systemic manifestations of MFS (Matt et al., 2009). In summary, all of these observations suggested that haploinsufficiency may be a critical determinant for the onset of MFS in mice.

To gain further insight into physiopathology and the effect of mutant FBN1 on the assembly and stability of microfibrils, Charbonneau et al. (2010) generated two knock-in mouse lines, GT- 8 and H1 $\Delta$. H1 $\Delta$ mice have an in-frame deletion of the first hydride domain (H1) in the Fbnl gene. This domain has 


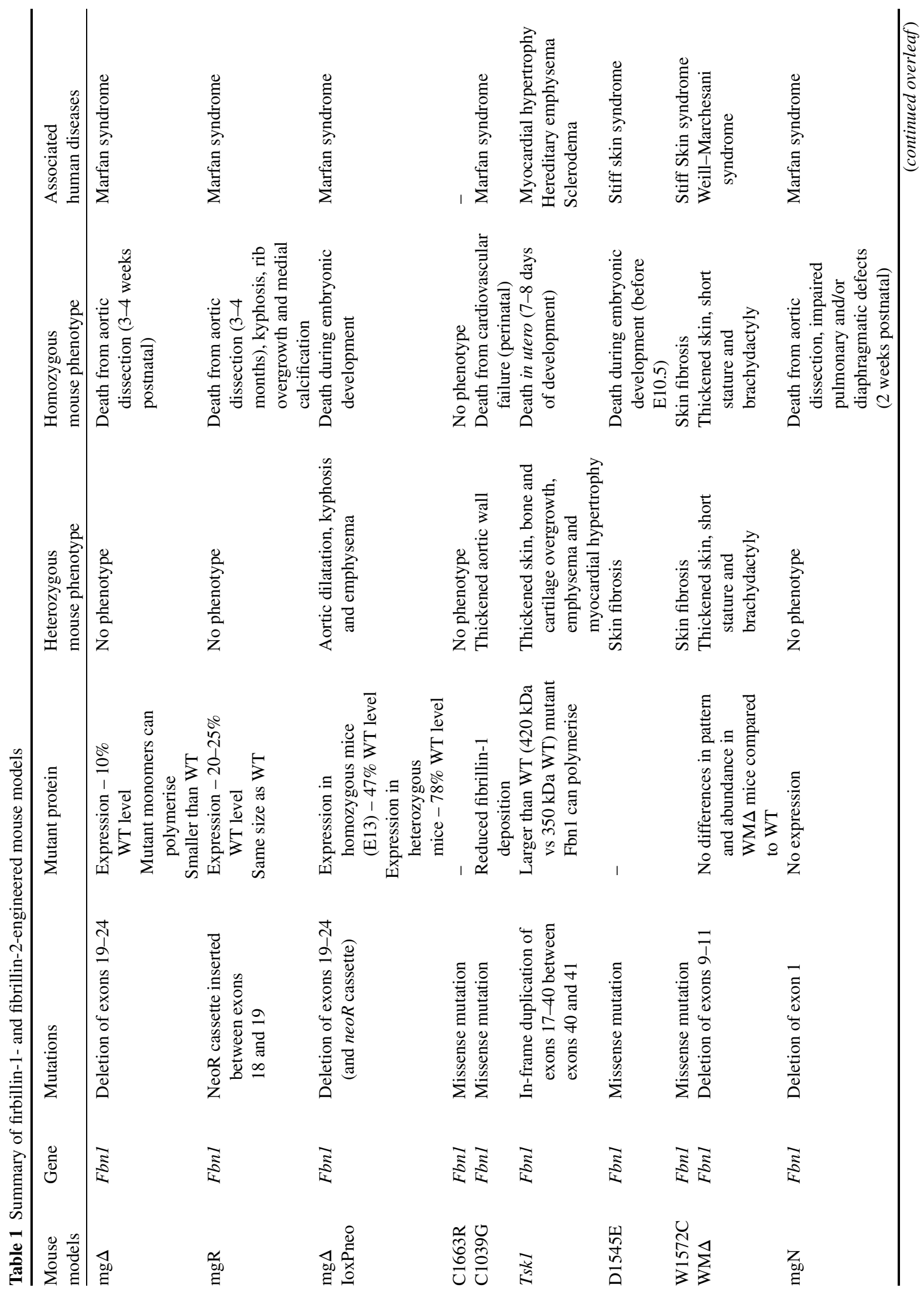




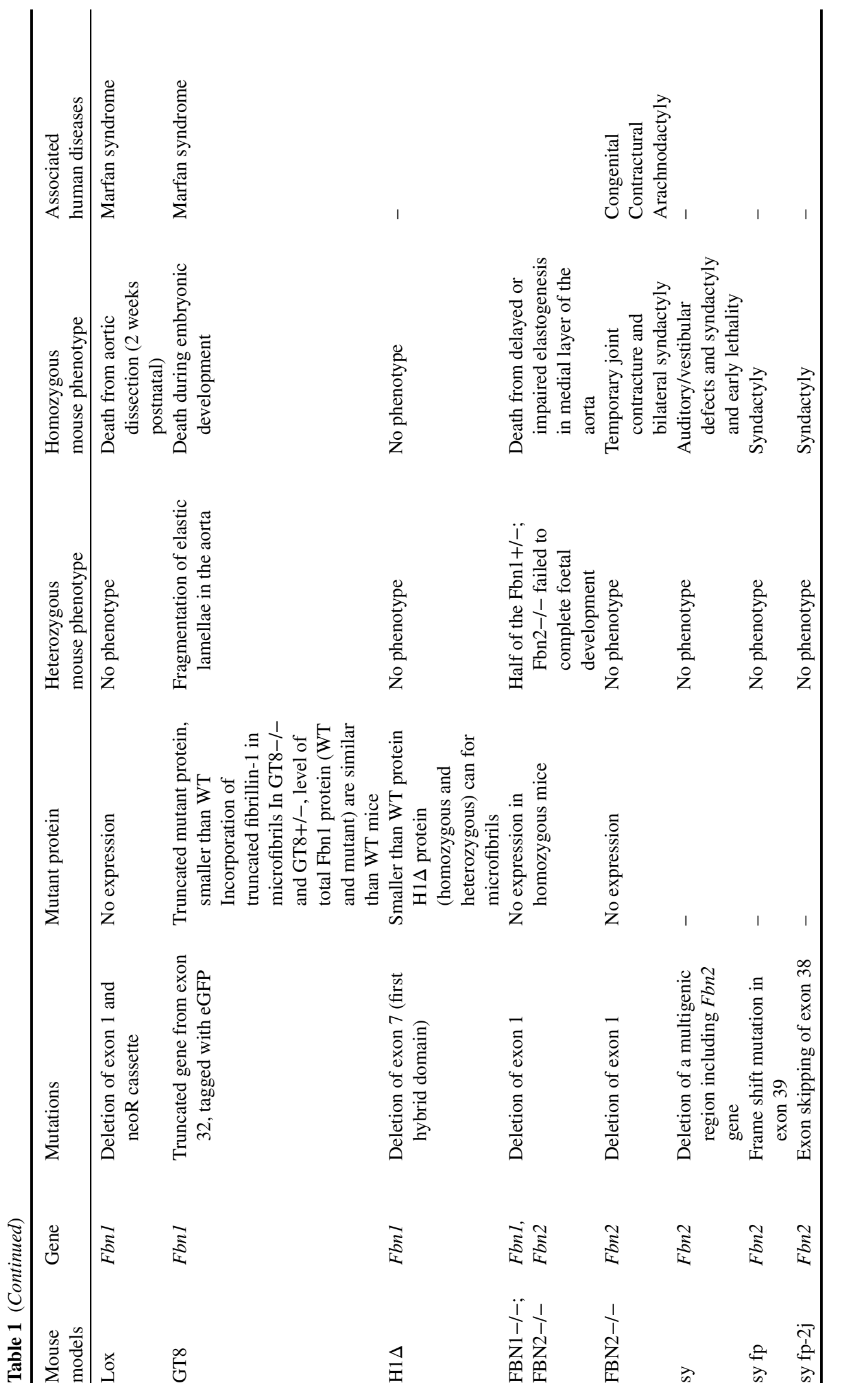


been shown to be involved in LTBPs and FBN1 interactions (Ono et al., 2009). H1s mice showed no abnormal phenotype or alteration of microfibril function and structure. In the GT-8 line, the Fbnl gene was truncated (from exon 32 until the end) and tagged with enhanced green fluorescent protein (eGFP). The truncated FBN1 was secreted like the wild-type protein and assembled in microfibrils. Homozygous mice were not viable, whereas in heterozygous mice, fragmentation of elastic lamellae in the aorta was seen. Therefore, truncated FBN1 did not interfere with microfibril assembly but exerted a dominant negative effect on the stability of microfibrils.s

Apart from MFS, mutations in the Fbn1 gene have been associated with other pathologies. The first mutation described was the Thight skin (Tsk1) mutation, a spontaneous autosomal dominant mutation in an inbred mouse strain. Tsk1 mice harbour in-frame duplication of exons 17-40 of Fbnl gene, between exons 40 and 41 , resulting in a larger Fbn1 protein. Homozygous Tsk1 were not viable, embryos died at 7-8 days of development, whereas heterozygous mice $(T s k 1 /+)$ had a normal life span. However, $T s k 1 /+$ mice had thickened skin, bone and cartilage overgrowth, lung emphysema and myocardial hypertrophy. On a histopathological level, Tsk1/+ mice display excessive accumulation of microfibrils, collagens and glycosaminoglycans in skin, heart and lungs, mimicking molecular abnormalities seen in patients with systemic sclerosis or scleroderma. Therefore, $T s k 1$ mice provide an opportunity to investigate tissue fibrosis pathogenesis at a molecular level. Moreover, Tsk1 mouse model has been used to study several other human diseases such as myocardial hypertrophy and hereditary emphysema.

As heterozygous missense mutations in Fbnl gene, all localised in an integrin-binding domain, have been described in SSS, two knock-in mice, W1572C and D1545E were engineered (Hubmacher et al., 2006). Both mutations were located in the integrin-binding domain causing an obligate loss of the interaction between Fbn1 and integrins to determine whether this loss is sufficient to cause skin fibrosis. Heterozygous mice in both strains showed SSS features including increased deposition of collagen and decreased subcutaneous fat. D1545E homozygous mice (D1545E-/-) were not viable, whereas W1572C homozygous mice were viable and showed earlier onset of skin fibrosis, compared to heterozygous mice. It should be noted that, 12-week treatment using integrin-activating antibodies rescued SSS phenotypes in SSS mouse models. All of these observations suggest that loss of integrin and FBN1 interaction is sufficient to initiate the onset of skin fibrosis, highlighting potential therapeutic strategies.

The autosomal dominant form of WMS is caused by mutations in the Fbnl gene (Faivre, 2003). Sengle et al. (2012) identified a novel mutation in a family with WMS, a deletion of exons 9-11 encoding three domains that act as binding sites for several proteins such as ADAMTSL proteins. Then, they replicated this mutation in the mouse to understand how FBN1 plays a role in connective tissue. WM $\Delta$ mutant mice, both heterozygous and homozygous, harboured a thickened skin associated with abnormalities in FBN1 microfibril structures, increased accumulation of microfibrils and reduced long bones normalised after 5 months of age. These mouse models allowed Sengle et al. (2012) to demonstrate that modulations of fibrillin-rich microfibril scaffolds could influence local tissue microenvironment and that FBN1 plays a role in skin homeostasis.

Finally, to better understand the role of fibrillins in embryonic development and especially in organogenesis, two mouse models have been created, both lacking $F b n l$ gene expression: mgN mice, in which exon 1 of Fbnlgene was deleted and replaced by a neoR cassette (Hubmacher et al., 2006) and FbnI ${ }^{\mathrm{CMV}}-\mathrm{I}-$ mice, in which both exon 1 and the neoR cassette were deleted (Cook et al., 2012). Homozygous mice from both strains died 2 weeks after birth owing to ruptured aortic aneurysm, impaired pulmonary function and/or diaphragmatic collapse. Analysis of their aorta showed disorganised elastic lamellar units in the medial layer. In summary, it suggested that FBN1 is involved in tissue homeostasis and also Cook et al. demonstrated that Fbn $1^{\text {Lox }}$ - mice were the adequate model to study tissue- and stage-specific role of FBN1 microfibrils, and so yielding mechanistic insights into MFS pathogenesis. Moreover, Cook et al. studied the consequences of combined deficiency of FBN1 and FBN2 (Fbn1-/-; Fbn2-/-). They showed that embryos were not viable and displayed a more severe cardiovascular phenotype than Fbn1-/- mice, supporting the idea that FBN2 is required for embryonic development. Surprisingly, mice completely lacking FBN2 display a mild phenotype (Carta et al., 2006). Indeed, homozygous mice (Fbn2-l-) showed temporary joint contractures which are clinical symptoms seen in patients with CAA. Fbn2-/- mice also display bilateral syndactyly and microfibrillar network disorganisation. It should be noted that Fbn2 gene mutation is not always associated with microfibrillar network disorganisation. Shaker-with-syndactylysm (sy) is a radiation-induced mutation causing a deletion of a multigenic region of chromosome 18, including the Fbn2 gene. This mutation results in auditory/vestibular defects, syndactyly and early lethality. Two alleles of $s y$ have been identified, $s y^{\text {fp }}$ (frameshift mutation in exon 39) and $s y^{\mathrm{fp}-2 \mathrm{~J}}$ (exon skipping of exon38). Both are due to spontaneous mutations and are also associated with syndactyly but neither of these mice lines displays abnormalities in the microfibrillar network. To this day, no similar phenotypes have been associated with FBN2 mutations in man.

In conclusion, fibrillin mouse models provided insights into the functional roles of Fbn1 and Fbn2 in development and tissue homeostasis as well as into the pathogenesis and the mechanisms involved in fibrillinopathies, allowing development and adjustment of therapeutic strategies.

\section{Conclusion}

Since the first description of fibrillins in the late 1980s, their role not only as major structural components of the ECM but also as regulators of the bioavailability of signalling molecules has been discovered and well investigated. The on-going description of the fibrillinopathies has provided a major contribution to this knowledge, highlighting specific aspects of fibrillin metabolism and relationship to other ECM proteins, to signalling mechanisms and the importance of cross-talk with vascular smooth muscles cells in the aortic wall. The next step will be reached when the genetic modifiers responsible for the wide clinical variability 
of the fibrillinopathies are identified. This will provide further insight into other aspects of fibrillin biology.

\section{Related Articles}

\section{Marfan Syndrome}

Molecular Genetics of Aortic Aneurysms and Aortic Dissections

\section{References}

Aubart M, Gross M-S, Hanna N, et al. (2015) The clinical presentation of Marfan syndrome is modulated by expression of wild-type FBN1 allele. Human Molecular Genetics 24: 2764-2770.

Barbier M, Gross M-S, Aubart M, et al. (2014) MFAP5 loss-offunction mutations underscore the involvement of matrix alteration in the pathogenesis of familial thoracic aortic aneurysms and dissections. American Journal of Human Genetics 95: 736-743.

Beighton P, de Paepe A, Danks D, et al. (1988) International nosology of heritable disorders of connective tissue, Berlin, 1986. American Journal of Medical Genetics 29: 581-594.

Biggin A, Holman K, Brett M, Bennetts B and Adès L (2004) FBN1 mutations in patients with Marfan syndrome or a related fibrillinopathy. Human Mutation 23: 99.

Boileau C, Guo D-C, Hanna N, et al. (2012) TGFB2 mutations cause familial thoracic aortic aneurysms and dissections associated with mild systemic features of Marfan syndrome. Nature Genetics 44 : 916-921.

Callewaert BL, Loeys BL, Ficcadenti A, et al. (2009) Comprehensive clinical and molecular assessment of 32 probands with congenital contractural arachnodactyly: report of 14 novel mutations and review of the literature. Human Mutation 30: 334-341.

Carta L, Pereira L, Arteaga-Solis E, et al. (2006) Fibrillins 1 and 2 perform partially overlapping functions during aortic development. Journal of Biological Chemistry 281: 8016-8023.

Charbonneau NL, Carlson EJ, Tufa S, et al. (2010) In vivo studies of mutant fibrillin-1 microfibrils. Journal of Biological Chemistry 285: 24943-24955.

Collod-Béroud G, Béroud C, Adès L, et al. (1997) Marfan database (second edition): software and database for the analysis of mutations in the human FBN1 gene. Nucleic Acids Research 25: 147-150.

Cook JR, Smaldone S, Cozzolino C, et al. (2012) Generation of Fbn 1 conditional null mice implicates the extracellular microfibrils in osteoprogenitor recruitment. Genesis 50: 635-641.

Corson GM, Chalberg SC, Dietz HC, et al. (1993) Fibrillin binds calcium and is coded by cDNAs that reveal a multidomain structure and alternatively spliced exons at the $5^{\prime}$ end. Genomics 17: 476-484.

De Paepe A, Devereux RB, Dietz HC, et al. (1996) Revised diagnostic criteria for the Marfan syndrome. American Journal of Medical Genetics 62: 417-426.

Détaint D, Faivre L, Collod-Beroud G, et al. (2010) Cardiovascular manifestations in men and women carrying a FBN1 mutation. European Heart Journal 31: 2223-2229.

Dietz HC, Cutting GR, Pyeritz RE, et al. (1991) Marfan syndrome caused by a recurrent de novo missense mutation in the fibrillin gene. Nature 352: 337-339.
Downing AK, Knott V, Werner JM, et al. (1996) Solution structure of a pair of calcium-binding epidermal growth factor-like domains: implications for the Marfan syndrome and other genetic disorders. Cell 85: 597-605.

Faivre L (2003) In frame fibrillin-1 gene deletion in autosomal dominant Weill-Marchesani syndrome. Journal of Medical Genetics 40: 34-36.

Faivre L, Collod-Beroud G, Callewaert B, et al. (2009) Pathogenic FBN1 mutations in 146 adults not meeting clinical diagnostic criteria for Marfan syndrome: further delineation of type 1 fibrillinopathies and focus on patients with an isolated major criterion. American Journal of Medical Genetics Part A 149A: 854-860.

Faivre L, Collod-Beroud G, Loeys BL, et al. (2007) Effect of mutation type and location on clinical outcome in 1,013 probands with Marfan syndrome or related phenotypes and FBN1 mutations: an international study. American Journal of Human Genetics 81: 454-466.

Gibson MA, Hatzinikolas G, Kumaratilake JS, et al. (1996) Further characterization of proteins associated with elastic fiber microfibrils including the molecular cloning of MAGP-2 (MP25). Journal of Biological Chemistry 271: 1096-1103.

Giltay R, Timpl R and Kostka G (1999) Sequence, recombinant expression and tissue localization of two novel extracellular matrix proteins, fibulin-3 and fibulin-4. Matrix Biology 18: 469-480.

Handford PA, Mayhew M, Baron M, et al. (1991) Key residues involved in calcium-binding motifs in EGF-like domains. Nature 351: $164-167$.

Hennekam RCM (2005) Severe infantile Marfan syndrome versus neonatal Marfan syndrome. American Journal of Medical Genetics Part A 139: 1.

Howarth R, Yearwood C, Harvey JF (2007) Application of dHPLC for mutation detection of the fibrillin-1 gene for the diagnosis of Marfan syndrome in a National Health Service Laboratory. Genetic Testing 11: 146-152.

Hubmacher D, Tiedemann K and Reinhardt DP (2006) Fibrillins: from biogenesis of microfibrils to signaling functions. Current Topics in Developmental Biology 75: 93-123.

Janssens K, Vanhoenacker F, Bonduelle M, et al. (2006) CamuratiEngelmann disease: review of the clinical, radiological, and molecular data of 24 families and implications for diagnosis and treatment. Journal of Medical Genetics 43: 1-11.

Jacquinet A, Verloes A, Callewaert B, et al. (2014) Neonatal progeroid variant of Marfan syndrome with congenital lipodystrophy results from mutations at the $3^{\prime}$ end of FBN1 gene. European Journal of Medical Genetics 57 (5): 230-234.

Jensen SA, Robertson IB and Handford PA (2012) Dissecting the fibrillin microfibril: structural insights into organization and function. Structure 20: 215-225.

Judge DP, Biery NJ, Keene DR, et al. (2004) Evidence for a critical contribution of haploinsufficiency in the complex pathogenesis of Marfan syndrome. Journal of Clinical Investigation 114: 172-181. Kielty CM, Sherratt MJ, Marson A and Baldock C (2005) Fibrillin microfibrils. Advances in Protein Chemistry 70: 405-436.

Lack J, O'Leary JM, Knott V, et al. (2003) Solution structure of the third TB domain from LTBP1 provides insight into assembly of the large latent complex that sequesters latent TGF-beta. Journal of Molecular Biology 334: 281-291.

Le Goff C, Mahaut C, Wang LW, et al. (2011) Mutations in the TGF $\beta$ binding-protein-like domain 5 of FBN1 are responsible for acromicric and geleophysic dysplasias. American Journal of Human Genetics 89: 7-14. 
Le Goff C and Cormier-Daire V (2012) From tall to short: the role of TGF $\beta$ signaling in growth and its disorders. American Journal of Medical Genetics Part C: Seminars in Medical Genetics 160C: 145-153.

Le Goff C, Mahaut C, Abhyankar A, et al. (2012) Mutations at a single codon in Mad homology 2 domain of SMAD4 cause Myhre syndrome. Nature Genetics 44: 85-88.

Loeys BL, Chen J, Neptune ER, et al. (2005) A syndrome of altered cardiovascular, craniofacial, neurocognitive and skeletal development caused by mutations in TGFBR1 or TGFBR2. Nature Genetics 37: 275-281.

Loeys BL, Gerber EE, Riegert-Johnson D, et al. (2010a) Mutations in fibrillin-1 cause congenital scleroderma: stiff skin syndrome. Science Translational Medicine 2: 23ra20-23ra20.

Loeys BL, Dietz HC, Braverman AC, et al. (2010b) The revised Ghent nosology for the Marfan syndrome. Journal of Medical Genetics 47 (7): 476-485.

Low FN (1962) Microfibrils: fine filamentous components of the tissue space. Anatomical Record 142: 131-137.

Matt P, Schoenhoff F and Habashi J (2009) Circulating transforming growth factor - in Marfan syndrome. Circulation 120: 526-532.

McCalmont TH and Gilliam AE (2012) A subcutaneous lattice-like array of thick collagen is a clue to the diagnosis of stiff skin syndrome. Journal of Cutaneous Pathology 39 (2-4): 1.

McKusick VA (1955) The cardiovascular aspects of Marfan's syndrome: a heritable disorder of connective tissue. Circulation 11: 321-342.

Mizuguchi T, Collod-Beroud G, Akiyama T, et al. (2004) Heterozygous TGFBR2 mutations in Marfan syndrome. Nature Genetics $\mathbf{3 6}$ : 855-860.

Morris SA, Orbach DB, Geva T, et al. (2011) Increased vertebral artery tortuosity index is associated with adverse outcomes in children and young adults with connective tissue disorders. Circulation 124: 388-396.

Ono RN, Sengle G, Charbonneau NL, et al. (2009) Latent transforming growth factor -binding proteins and fibulins compete for fibrillin-1 and exhibit exquisite specificities in binding sites. Journal of Biological Chemistry 284: 16872-16881.

Pereira L, Lee SY, Gayraud B, et al. (1999) Pathogenetic sequence for aneurysm revealed in mice underexpressing fibrillin-1. Proceedings of the National Academy of Sciences 96: 3819-3823.

Putnam EA, Zhang H, Ramirez F and Milewicz DM (1995) Fibrillin-2 (FBN2) mutations result in the Marfan-like disorder, congenital contractural arachnodactyly. Nature Genetics 11: 456-458.

Quondamatteo F, Reinhardt DP, Charbonneau NL, et al. (2002) Fibrillin-1 and fibrillin-2 in human embryonic and early fetal development. Matrix Biology 21: 637-646.

Ramirez F and Sakai LY (2010) Biogenesis and function of fibrillin assemblies. Cell and Tissue Research 339: 71-82.

Regalado ES and Milewicz DM (2015) Molecular genetics of aortic aneurysms and aortic dissections. In: eLS. DOI: 10.1002/9780470015902.a0024366.

Robinson PN (2006) Marfan syndrome. In: eLS. DOI: 10.1038/npg.els.0006087.

Sakai LY, Keene DR, Glanville RW and Bächinger HP (1991) Purification and partial characterization of fibrillin, a cysteine-rich structural component of connective tissue microfibrils. Journal of Biological Chemistry 266: 14763-14770.
Samadi A, Detaint D, Roy C, et al. (2012) Surgical management of patients with Marfan syndrome: evolution throughout the years. Archives of Cardiovascular Diseases 105: 84-90.

Sengle G, Tsutsui K, Keene DR, et al. (2012) Microenvironmental regulation by fibrillin-1. PLoS Genetics 8: e1002425.

Sengle G and Sakai LY (2015) The fibrillin microfibril scaffold: a niche for growth factors and mechanosensation? Matrix Biology 47: 3-12.

Sakai H, Visser R, Ikegawa S, et al. (2006) Comprehensive genetic analysis of relevant four genes in 49 patients with Marfan syndrome or Marfan-related phenotypes. American Journal of Medical Genetics Part A 140: 1719-1725.

Singleton AC, Mitchell AL, Byers PH, et al. (2005) Bovine model of Marfan syndrome results from an amino acid change (c.3598G > A, p.E1200K) in a calcium-binding epidermal growth factor-like domain of fibrillin-1. Human Mutation 25: 348-352.

Siracusa LD, McGrath R, Ma Q, et al. (1996) A tandem duplication within the fibrillin 1 gene is associated with the mouse tight skin mutation. Genome Research 6: 300-313.

Stheneur C, Faivre L, Collod-Béroud G, et al. (2011) Prognosis factors in probands with an FBN1 mutation diagnosed before the age of 1 year. Pediatric Research 69: 265-270.

Van de Laar IMBH, Oldenburg RA, Pals G, et al. (2011) Mutations in SMAD3 cause a syndromic form of aortic aneurysms and dissections with early-onset osteoarthritis. Nature Genetics 43: 121-126.

Wang M, Clericuzio CL and Godfrey M (1996) Familial occurrence of typical and severe lethal congenital contractural arachnodactyly caused by missplicing of exon 34 of fibrillin-2. American Journal of Human Genetics 59: 1027-1034.

\section{Further Reading}

Gallo EM, Loch DC, Habashi JP, et al. (2014) Angiotensin II-dependent TGF- $\beta$ signaling contributes to Loeys-Dietz syndrome vascular pathogenesis. Journal of Clinical Investigation 124: 448-460.

Gerber EE, Gallo EM, Fontana SC, et al. (2013) Integrin-modulating therapy prevents fibrosis and autoimmunity in mouse models of scleroderma. Nature 503: 126-130.

Holm TM, Habashi JP, Doyle JJ, et al. (2011) Noncanonical TGF $\beta$ signaling contributes to aortic aneurysm progression in Marfan syndrome mice. Science 332: 358-361.

Lee SSJ, Knott V, Jovanović J, et al. (2004) Structure of the integrin binding fragment from fibrillin-1 gives new insights into microfibril organization. Structure 12: 717-729.

Lin G, Tiedemann K, Vollbrandt T, et al. (2002) Homo- and heterotypic fibrillin-1 and -2 interactions constitute the basis for the assembly of microfibrils. Journal of Biological Chemistry 277: 50795-50804.

Sechler JL, Rao H, Cumiskey AM, et al. (2001) A novel fibronectin binding site required for fibronectin fibril growth during matrix assembly. Journal of Cell Biology 154: 1081-1088.

Zhang H, Hu W and Ramirez F (1995) Developmental expression of fibrillin genes suggests heterogeneity of extracellular microfibrils. Journal of Cell Biology 129: 1165-1176. 